This item was submitted to Loughborough's Research Repository by the author.

Items in Figshare are protected by copyright, with all rights reserved, unless otherwise indicated.

\title{
Ripplocations in layered materials: Sublinear scaling and basal climb
}

\section{PLEASE CITE THE PUBLISHED VERSION}

https://doi.org/10.1103/physrevb.103.195436

\section{PUBLISHER}

American Physical Society (APS)

\section{VERSION}

VoR (Version of Record)

\section{PUBLISHER STATEMENT}

The published version is also available on the publisher's website at https://doi.org/10.1103/physrevb.103.195436.

\section{LICENCE}

CC BY-NC-ND 4.0

\section{REPOSITORY RECORD}

McHugh, James, Paul Mouratidis, and Kenny Jolley. 2021. "Ripplocations in Layered Materials: Sublinear Scaling and Basal Climb". Loughborough University. https://hdl.handle.net/2134/16607354.v1. 


\title{
Ripplocations in layered materials: Sublinear scaling and basal climb
}

\author{
James G. McHugh $\odot,{ }^{*}$ Pavlos Mouratidis $\odot$, and Kenny Jolley $\odot$ \\ Department of Chemistry, Loughborough University, Epinal Way, Loughborough LE11 3TU, United Kingdom
}

(Received 18 December 2020; revised 24 March 2021; accepted 10 May 2021; published 24 May 2021)

\begin{abstract}
The ripplocation is a crystallographic defect which is unique to layered materials, combining nanoscale delamination with the crystallographic slip of a basal dislocation. Here, we have studied basal dislocations and ripplocations, in single and multiple van der Waals layers, using analytical and computational techniques. Expressions for the energetic and structural scaling factors of surface ripplocations are derived, which are in close correspondence to the physics of a classical carpet ruck. Our simulations demonstrate that the lowest-energy structure of dislocation pileups in layered materials is the ripplocation, while large dislocation pileups in bulk graphite demonstrate multilayer delamination, curvature, and voids. This can provide a concise explanation for the large volumetric expansion seen in irradiated graphite.
\end{abstract}

DOI: 10.1103/PhysRevB.103.195436

\section{INTRODUCTION}

It has long been understood that the plastic deformation of solids proceeds primarily through the nucleation of linear defects, called dislocations, which locally accommodate crystallographic slip. The slipped and unslipped crystal regions on either side of a dislocation line are related through translation by a lattice vector, which is a topological property of that dislocation, called its Burgers vector, b. The dynamics of dislocations are dominated by Frank's rule, which dictates that the energy of a dislocation is quadratic in the Burgers vector, $E \propto \mathbf{b}^{2}$. This causes perfect dislocations to dissociate into partials, with Burgers vector of less than a full lattice translation [1-3].

Recently, distinct behavior has been observed in layered materials. Surface ripples with well-defined crystallographic character comprising sharp, localized folds between regions which are slipped relative to one another by an in-plane lattice vector have been observed at the (0001) surface of exfoliated $\mathrm{MoS}_{2}$ samples and graphite nanoplatelets [4,5]. These defects combine the properties of edge dislocations and delamination, and have been termed ripplocations. Extensive spherical nanoindentation experiments have demonstrated that the deformation behavior of a wide variety of layered materials proceeds not through the basal dislocation, but through a distinct and reversible mechanism, which has been attributed to the formation of ripplocations [6]. Reversible arrays of ripplocation boundaries which can evolve into kink bands under increasing damage are believed to constitute the initial states of material failure in layered materials. Ripplocations are now believed to occur on a variety of length scales, accounting for material deformation in substances as diverse as playing cards and steel sheets to phyllosilicates in the lithosphere [7-9]. Additionally, surface wrinkles on homogeneous substrates necessarily have edge dislocation character below a critical

*j.g.mchugh@lboro.ac.uk length [10], hence the study of ripplocations will also give further insight into the wrinkling behavior of 2D materials, which can substantially modify the physical properties of adsorbed monolayers [11-14].

Ripplocations occur both epitaxially and in the bulk of layered materials. In the context of irradiated graphite, the bulk ripplocation or "ruck and tuck" mechanism of dislocation pileup represents the first proposal of deformation due to delamination in the bulk of layered solids, and is likely to be particularly important in explaining many of the properties of dimensional change in highly irradiated highly oriented pyrolytic graphite (HOPG) samples. However, while this type of bulk ripplocation has been found in experimental transmission electron microscopy (TEM) images of irradiated graphite [8,15-20], it is a relatively rare defect. Distinct behavior, including delamination and buckling spread across many layers and two-dimensional kink band networks [21,22], has also been observed. This motivates further, systematic studies of multilayer and two-dimensional dislocation configurations.

In contrast to the classical dislocation, ripplocations exhibit a sublinear energy scaling as a function of Burgers vector, $E \propto \mathbf{b}^{\beta}$, where $\beta<1$. This ensures that ripplocations composed of multiple Burgers vector become increasingly energetically favorable as a function of extra material, as $\left|\mathbf{b}_{1}+\mathbf{b}_{2}\right|^{\beta}<\left|\mathbf{b}_{1}\right|^{\beta}+\left|\mathbf{b}_{2}\right|^{\beta}$. While this sublinearity has been well established, there is reasonable variation in the literature with reported values lying in the range $\beta \approx 0.3-0.45$ $[4,23,24]$. Additionally, while there have been computational studies of ripplocations, no rigorous comparison has been made between them and the corresponding dislocation cores.

In this work, we have applied analytical and computational techniques which accurately model the interlayer friction between van der Waals layers to calculate the energetic and structural scaling factors of single and multilayer surface and bulk ripplocations. In van der Waals materials the interlayer friction is encapsulated in the $\gamma$-surface energy to slide adjacent layers along different crystallographic directions [25-29]. This is essential in accurately modeling these 
defects, as it is the resistance to sliding against adjacent layers that stabilizes basal dislocations and ripplocations. Our simulations of dislocation pileups in large systems demonstrate that the locally buckled ripplocation structure is the preferred mode of deformation with increasing additional material in the pileup.

\section{METHODS}

Density functional theory (DFT) calculations have been performed using plane-wave basis Kohn-Sham states as implemented in the Quantum ESPRESSO package [30,31]. We have employed Vanderbilt ultrasoft pseudopotentials parametrized according to the local density approximation (LDA). While it is often assumed that the inclusion of gradient terms in the generalized gradient approximation (GGA) makes it a better approximation than the simpler LDA, in some cases LDA performs significantly better than GGA. Notably, the LDA provides a more accurate approximation to the interlayer van der Waals interaction in comparison to the GGA [32], when benchmarked against higher levels of theory such as quantum Monte Carlo (QMC) calculations $[33,34]$. The LDA also provides an acceptable approximation to the dispersion interaction for weakly bonded materials when compared to van der Waals-corrected GGA functionals, producing a somewhat larger than average error for binding energies and lattice constants [35]. For graphite in particular, the LDA compares relatively well to vdW-corrected GGA in predicting the $a$ and $c$ lattice constants, bulk moduli [36], and cohesive [37] and exfoliation energies [38,39], such that functional choice is overall unlikely to qualitatively affect our results.

Electronic wave functions were expanded in a plane-wave basis with cutoffs of $E_{\text {cut }}=40 \mathrm{Ry}$. A $\Gamma$-centered $1 \times 5 \times 1$ $k$-point grid is found to sample the Brillouin zone with sufficient accuracy, giving total energy convergence to within $0.1 \mathrm{meV} /$ atom for all structures. Molecular dynamics (MD) simulations have been performed in LAMMPS [40], using the hybrid neural network (hNN) potential for multilayer graphene systems developed by Wen and Tadmor [41,42]. We have taken care to assess the suitability of the hNN potential and a variety of interlayer potentials $[26,28,43,44]$ in capturing the behavior of dislocations and ripplocations (for additional details see Supplemental Material [45] Figs. S1 and S2).

\section{SURFACE RIPPLOCATIONS}

Surface ripplocations have been created through the insertion of additional rows of material to the top monolayer of a bilayer structure in a periodic ribbon geometry, which is extended along one crystallographic orientation. Atoms in the top layer are allowed to fully relax, while the bottom layer is held fixed by setting all forces to zero, to approximate a compressed monolayer on a substrate. This approach has been verified in comparison to a fully relaxed, multilayer graphite substrate using MD calculations, which produces very similar qualitative behavior, with a small quantitative difference which we attribute to relaxation of the multilayer substrate (for more details see Supplemental Material [45] Figs. S3 and S4).

The amount of additional material is quantified using the parameter $n$, denoting a Burgers vector of multiple lattice translations. Along the zigzag direction this corresponds to an edge dislocation $(n=1)$ or superdislocation $(n>1), \mathbf{b}_{\mathbf{z z}}=$ $n \times[1 \overline{2} 10]$. Along the armchair direction $\mathbf{b}_{\mathbf{a c}}=n \times[\overline{1} 100]$, and all edge dislocations are superdislocations. Thus, $n$ quantifies the number of edge dislocations in the superdislocation pileup, and is the characteristic property of a ripplocation defect.

DFT structural relaxations have been performed for zigzag surface ripplocations, $\mathbf{b}=n \times[1 \overline{2} 10]$, in graphene, hexagonal boron nitride $(\mathrm{hBN})$, and $\mathrm{MoS}_{2}$. While the initial configuration is that of a superdislocation, with a well-defined Burgers vector, upon relaxation the large initial in-plane strains are released by out-of-plane buckling. This gives close to equilibrium bond lengths across the ripplocation (see Supplemental Material [45] Figs. S5 and S6), and prior work has suggested that ripplocations do not possess a long-range Burgers vector [4,23].

Formation energies $E_{f}$ have been calculated from the optimized supercells as

$$
E_{f}=E_{\text {cell }}-N \epsilon_{\mathrm{BL}}
$$

where $E_{\text {cell }}$ is the energy of a defected bilayer, $\epsilon_{\mathrm{BL}}$ is the energy per atom of a perfect bilayer, and $N$ is the number of atoms. The height has been calculated as the distance between the flat part of the top layer and the ripple's peak, while the width was taken as the FWHM, both of which are shown schematically in Fig. 1(d). The formation energy as a function of $n$ is shown in Fig. 1(a) for all three materials, where we observe the expected sublinear dependence on $\mathbf{b}$, with similar scaling factors in the range $\beta \approx 0.37-0.41$ (see Supplemental Material [45] Fig. S7 for additional log-log plots which confirm the distinctive sublinear scaling factors).

Similarly, sublinear scaling is observed in the height and width, with consistent values for ripplocations in all three materials, as shown in Figs. 1(b) and 1(c). Further simulations of a graphene wrinkle on the $\mathrm{Cu}(100)$ surface (for additional details see Supplemental Material [45] Figs. S8 and S9) produce nearly identical structural scaling factors, which is highly suggestive that similar physics underlies both types of ripples.

\section{BASAL DISLOCATIONS}

The sublinear energy scaling guarantees that ripplocations will eventually become lower in energy than the corresponding dislocation structures, and that with increasing Burgers vector, the dislocation core will eventually buckle and release its strain in the out-of-plane direction. However, the precise transition point between these two defects is unclear. In order to gain more detailed insight into the energetics and dynamics of ripplocations, we now compare the corresponding properties of basal dislocations. The basal dislocation core in graphite and other layered materials is wide $(>10 \mathrm{~nm})$ [46-48] due to the weak interlayer interactions, and therefore difficult to simulate via DFT $[49,50]$. 

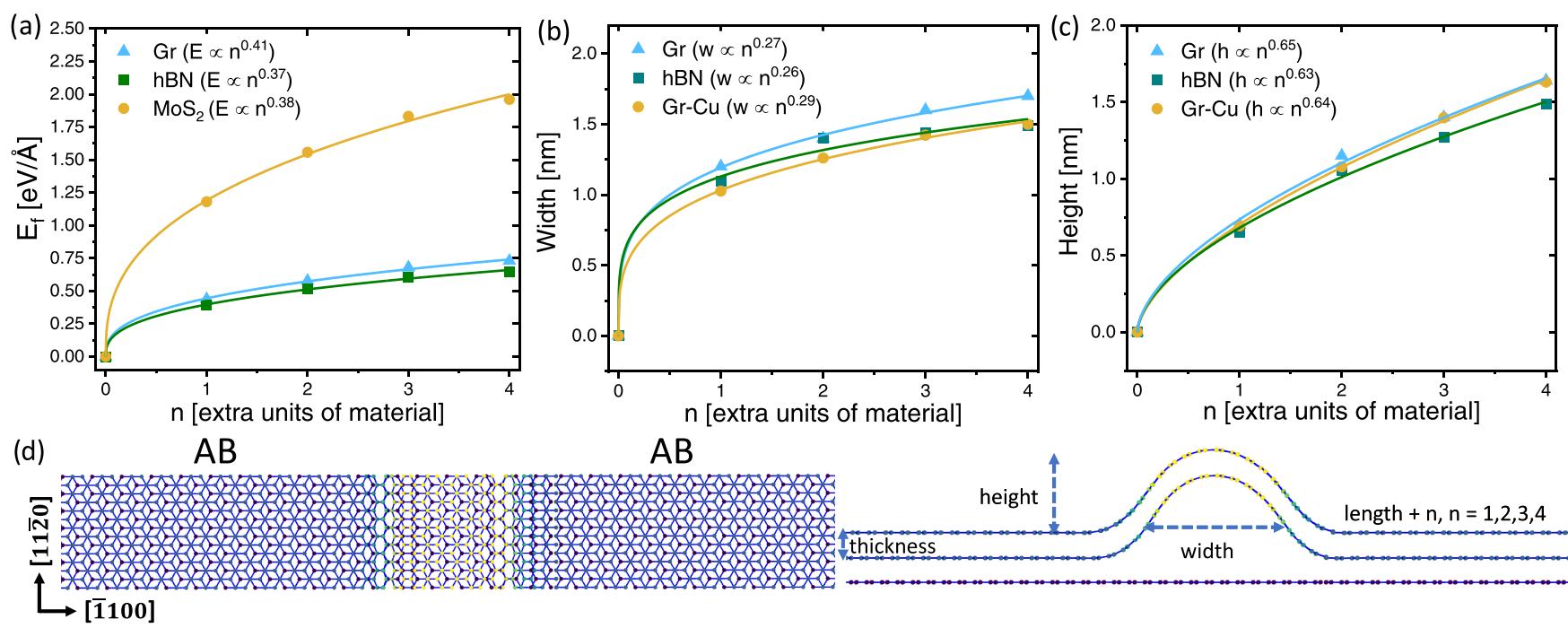

$A B$

FIG. 1. (a) Formation energy of ripplocations in epitaxial graphene, $\mathrm{MoS}_{2}$, and hBN sheets vs amount of additional material $n$. (b) Width and (c) height of ripplocations on epitaxial graphene on graphene, $\mathrm{hBN}$ on hBN, and graphene on the $\mathrm{Cu}(100)$ surface. (d) Schematic depiction of ripplocation configurations and associated structural properties as they have been calculated in this work.

To circumvent this, we have conducted MD simulations of large cells of up to $500 \mathrm{~nm}$ in length. In conducting MD simulations of interlayer defects in layered materials, it is of crucial importance to capture the interlayer friction or $\gamma$-surface energy. We have therefore taken particular care in comparing a variety of the available MD potentials to our DFT calculations. All of the potentials we consider agree qualitatively with our DFT results for the $\gamma$-surface energy $[26,28,41,49]$, as they are all fitted to quantum mechanical calculations of this property.

To further validate these potentials and compare to our DFT results, we have performed relaxation of surface ripplocations for increasing $n$, along both armchair and zigzag directions. Our results for the energetic and structural scaling factors using the hNN potential are consistent with DFT, as are all of the other potentials which produce a significant interlayer friction energy (for more details see Figs S10-S12). A full comparison of the scaling factors for all of the interlayer potentials considered, as well as the DFT results, is shown in Table I. We note that all of the computational methods predict highly sublinear energy scaling in agreement with DFT, and are generally within a relatively small range of values despite differences in choice of material, level of theory, and computational method. Furthermore, simulations of multilayer ripplocations [i.e., finite thickness, as shown schematically in Fig. 1(d)], undertaken using the hNN potential, also predict similar sublinear scaling factors.

In contrast, potentials which do not correctly capture the $\gamma$-surface energy are not able to correctly capture ripplocation properties with increasing cell size. For example, while the AIREBO potential is commonly used in studies of graphene and graphite, the $\gamma$-surface energy is significantly underestimated in comparison to DFT. This has immediate implications for the epitaxial properties of surface ripplocations. In particular, we find that this potential does not give consistent results (see Fig. 2). With increasing cell length, the ripple height decreases significantly, and for cells above approximately $55 \mathrm{~nm}$ ripplocations are not formed. Instead, the ripplocation ruck slides into the adjacent layers in a completely incommensurate manner (i.e., without forming basal dislocations).

TABLE I. Energy, width, and height scalings of surface ripplocations from different computational methods. Corresponding values for the best-fit value of the sublinearity factor $\alpha$ are shown in square brackets.

\begin{tabular}{|c|c|c|c|c|}
\hline Method & $\alpha_{\text {best fit }}$ & $E \propto b^{\beta(\alpha)}$ & $h \propto b^{\delta(\alpha)}$ & $w \propto b^{\gamma(\alpha)}$ \\
\hline Gravity & 1.0 & $5 / 7$ & $4 / 7$ & $1 / 7$ \\
\hline Gr (DFT) & 0.191 & $0.401[0.415]$ & $0.588[0.646]$ & $0.210[0.292]$ \\
\hline HBN (DFT) & 0.139 & $0.388[0.393]$ & $0.629[0.651]$ & $0.197[0.303]$ \\
\hline $\mathrm{MoS}_{2}(\mathrm{DFT})$ & 0.127 & $0.378[0.388]$ & $0.610[0.652]$ & $0.287[0.305]$ \\
\hline $\mathrm{KC}(\mathrm{MD})$ & 0.173 & $0.402[0.408]$ & $0.623[0.647]$ & $0.187[0.295]$ \\
\hline DRIP (MD) & 0.197 & $0.411[0.418]$ & $0.617[0.645]$ & $0.226[0.291]$ \\
\hline LP (MD) & 0.170 & $0.382[0.406]$ & $0.549[0.648]$ & $0.109[0.296]$ \\
\hline hNN (MD) & 0.098 & $0.382[0.413]$ & $0.679[0.646]$ & $0.223[0.293]$ \\
\hline $\mathrm{hNN} t=2$ & 0.111 & $0.408[0.381]$ & $0.759[0.654]$ & $0.271[0.309]$ \\
\hline $\mathrm{hNN} t=3$ & 0.133 & $0.391[0.391]$ & $0.736[0.674]$ & $0.287[0.304]$ \\
\hline $\mathrm{hNN} t=4$ & 0.127 & $0.388[0.388]$ & $0.765[0.674]$ & $0.305[0.306]$ \\
\hline
\end{tabular}




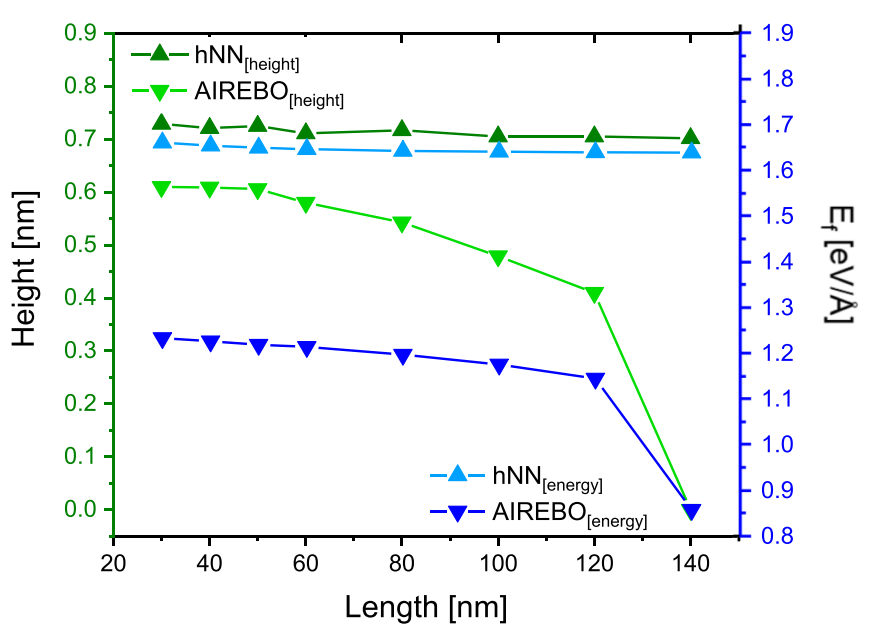

FIG. 2. Formation energy and height of the $n=1$ surface ripplocation using the AIREBO and $\mathrm{hNN}$ potentials.

Having verified the validity of our computational methods, we now compare the properties of ripplocations to the corresponding dislocation and superdislocation cores. The structures considered have an identical Burgers vector, i.e., amount of extra material, in the core region but are constrained to remain flat and the two defects are therefore directly comparable. Figures 3(c) and 3(d) compare the energy of ripplocations vs the corresponding superdislocation cores as a function of $n$, calculated using the $\mathrm{hNN}$ potential. It can be seen that at small $n$ for Burgers vector along both crystallographic directions, the lowest-energy structures are not localized ripples, but rather arrays of partial dislocations with net Burgers vector equal to the initial superdislocation Burgers vector, as shown in Fig. 3(a) and Fig. 3(b).

For the zigzag core this gives pairs of $\pm 60^{\circ}$ partials, $\mathbf{b}_{\mathbf{z z}}=$ $n \times 1 / 3[1 \overline{2} 10]=n \times(1 / 3[1 \overline{1} 00]+1 / 3[0 \overline{1} 10])$ for $n<3$, above which ripplocations are nucleated in preference to dislocation arrays. Along the armchair direction, the initial $n=1$ superdislocation dissociates into a sequence of four partial dislocations, a pair of $\pm 30^{\circ}$ partials and a pair of edge partials, $\mathbf{b}_{\mathbf{a c}}=n \times \sqrt{3}[10 \overline{1} 0]=n \times(\sqrt{3} / 6[1 \overline{1} 00]+$ $\sqrt{3} / 6[01 \overline{1} 0]+\sqrt{3} / 3[10 \overline{1} 0]+\sqrt{3} / 3[10 \overline{1} 0])$, and ripplocations are preferred for $n>1$. Overall, at relatively small Burgers vector we observe the onset of rippling and buckling in preference to in-plane strain accommodation for both crystallographic directions. This behavior is again consistent with that of a ruck in a classical inextensible material, where under an initial compression, a sheet will not buckle unless the ratio of the initial compression to the coefficient of static friction of the substrate interaction, here encapsulated in the $\gamma$ surface, is above a critical value [51].

\section{SCALING RELATIONS}

The near universality of the observed scaling law warrants further consideration. In prior work, the energetic and structural scaling factors for a ruck under the influence of gravity have been calculated [51,52]. Taking into account our prior observation that the bond strain across a surface ruck is generally very low (see Supplemental Material [45] Figs. S5 and S6), it is valid to consider a surface ripplocation as just such a ruck in an inextensible material. It is therefore (a)

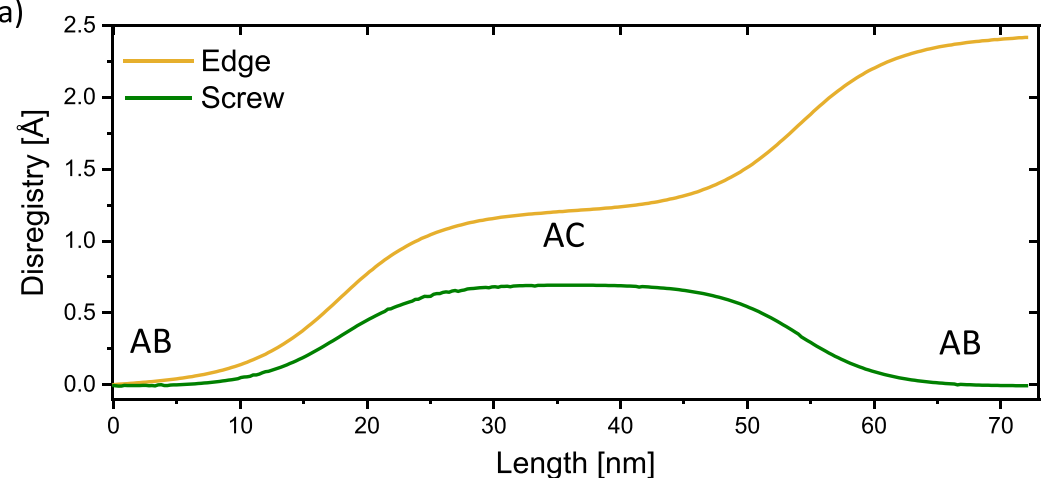

(b)

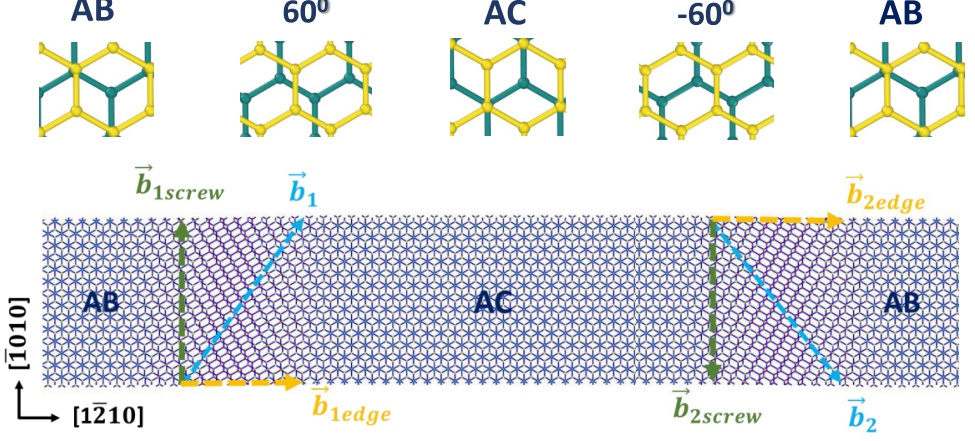

(c)

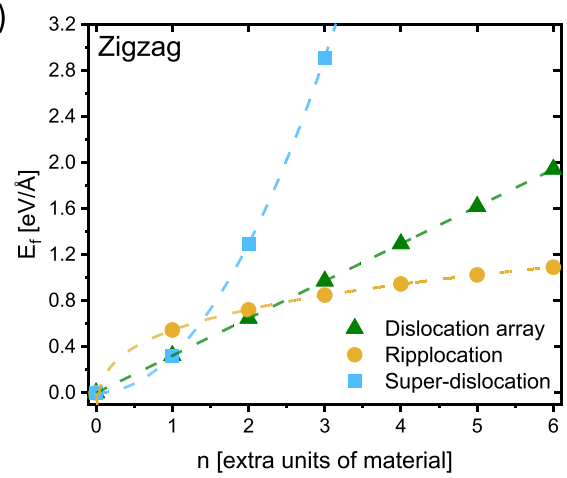

(d)

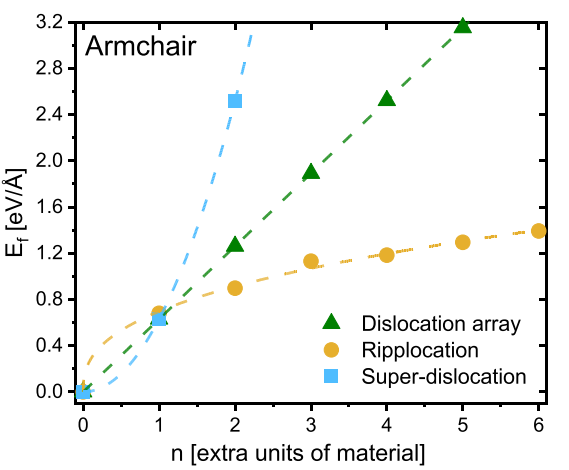

FIG. 3. (a) Disregistry calculated normal to the dislocation line (Edge) component and along the line (Screw) vs length of bilayer and (b) cell configuration and local stacking in a dislocated bilayer with two oppositely signed partial mixed dislocations. (c) Comparison of superdislocation, dislocation array, and ripplocation energies for Burgers vectors along the zigzag and (d) armchair directions. 
noteworthy that our computational values are quite different from these classical scalings (see Table I), particularly for the height and energy.

We therefore revisit and modify these scalings, by considering two energetic contributions to the core energy $[4,51,52]$. We emphasize that inextensibility is an important condition in the following derivation, as it implies that the membrane force [53] across the ruck is small, which allows us to ignore the contribution of strain to the ripple formation energy. This has been explicitly verified by calculating the strain contribution for the hNN model, which we find to be approximately $0.1 \mathrm{eV}$ and crucially is nearly constant as a function of $n$. The total energy of a ripplocation ruck is then taken to be

$$
E(w)=U_{e}(w)+U_{s}(w)=B \kappa^{2} w+V t h^{\alpha} w .
$$

The relevant energy terms comprise the elastic energy $U_{e}$ and the adhesion energy $U_{s}$, which is the energy acquired from the shifting and out-of-plane deformation across a ripple. The elastic energy is taken simply as $U_{e}=B \kappa^{2} w$ where $B$ is the elastic bending energy, $w$ is the width, and $\kappa$ is the net curvature across a ripple. The interfacial term encapsulates the adhesion energy across the core, which we take to be $V t h^{\alpha} w$ where $V$ is the interlayer adhesion energy of the absorbed layer to the substrate, $h$ is the height of the ruck, and $t$ is the monolayer thickness.

The most important modification of this expression with respect to the classical equation for an inextensible ruck is the incorporation of the sublinearity factor $\alpha$, which accounts for the deviation from classical behavior for van der Waals layers. We note that when $\alpha=1$ this corresponds to the classical rucking of a sheet under the influence of gravity. Making use of the relations $h=\sqrt{b w}$ and $\kappa=\frac{h}{w^{2}}$ for the arclength and curvature of a clamped elastica, the energy can be expressed as a function of width only, $E(w)=B b w^{-2}+V t b^{\alpha / 2} w^{1+\alpha / 2}$. We then solve for the equilibrium width by considering the derivative of the energy equation, Eq. (2), with respect to the defect width,

$$
\frac{d E(w)}{d w}=-\frac{2 B b}{w^{3}}+(1+\alpha / 2) V t(b w)^{\alpha / 2}=0 .
$$

This immediately leads to analytical forms for the scaling of ruck height and width as $w(b) \propto b^{\gamma(\alpha)}$ and $h(b) \propto b^{\delta(\alpha)}$, with

$$
\gamma(\alpha)=\frac{2-\alpha}{6+\alpha}
$$

and

$$
\delta(\alpha)=\frac{1}{2}+\frac{2-\alpha}{12+2 \alpha} .
$$

Replacing the height and width for these expressions in Eq. (2), the ruck energy as a function of width is then $E(w)=(B+t V) A(\alpha)^{\frac{2+\alpha}{++\alpha}} b^{1-(4-2 \alpha) /(6+\alpha)}$, where $A(\alpha)=$ $\frac{2 B}{t V(1+\alpha / 2)}$, hence leading to a sublinear energy scaling

$$
\beta(\alpha)=1-\frac{4-2 \alpha}{6+\alpha} .
$$

The sublinear scaling as a function of height can be understood as a consequence of the shorter range of the van der Waals interaction, which scales as a function of distance as $r^{-6}$, here incorporated approximately through the sublinearity factor $\alpha$. This leads to the fact that material closer to the substrate matters proportionally much more than that which is further away, so that the overall energy scales very slowly in the maximum height.

Notably, our calculations indicate very similar scaling factors across all materials and approximations. For example, our calculations indicate that the interlayer adhesion energy factor $V$ of $\mathrm{MoS}_{2}$ is around $50 \%$ higher than that of graphene, yet the calculated scalings remain in a very similar range, indicating that it is indeed the short-ranged behavior of the interlayer interaction which determines ruck properties, rather than the magnitude of the adhesion energy.

Thus, if the introduction of a sublinear term is an accurate model of the rippling across a ruck in a two-dimensional monolayer, all three properties will be consistently interrelated through a common sublinearity factor. Table I shows the calculated scaling of energy, height, and width for ripplocations in different materials and using different methods, as well as a "best fit" value of $\alpha$. This value minimizes the difference, $\epsilon$, between the analytical and computational values for all three scalings, $\epsilon=$ $\sqrt{\left(\beta_{\text {fit }}-\beta_{\text {comp }}\right)^{2}+\left(\delta_{\text {fit }}-\delta_{\text {comp }}\right)^{2}+\left(\gamma_{\text {fit }}-\gamma_{\text {comp }}\right)^{2}}$. The analytical expression produces an extremely good agreement with the computational sublinear energy dependence, with small errors in the height and width in the range of $0.05-0.1$. We attribute this small departure from the ideal behavior for the structural properties to the violation of the $h=\sqrt{b w}$ assumption for our van der Waals systems (see Supplemental Material [45] Fig. S13).

Overall, our calculations confirm that all three scalings are indeed closely related. Additionally, since we have only considered the bending and adhesion energies, this scaling behavior is immediately applicable to physisorbed monolayers on other substrates; this explains why our DFT simulation of the graphene $/ \mathrm{Cu}(100)$ surface interaction produces scalings which agree closely with the ripplocation results in Figs. 1(b) and $1(\mathrm{c})$.

\section{BULK}

We now discuss the behavior of bulk ripplocations in large cells. Cells containing bulk ripplocations are created similarly to surface ripplocations, where extra rows of material are inserted into a monolayer in a bulk cell. For small $n$, this again gives dislocation arrays and a linear energy dependence. Above a critical value of $n$, the formation energy is almost constant with additional material as shown in Fig. 4(b), and the folded-over bulk ripplocation, or "ruck and tuck" structure, is nucleated [54]. Accurate $\gamma$-surface energy is again paramount in attaining accurate defect structure, which for bulk ripplocations is reflected in the surrounding sheets, which have the lowest-energy Bernal stacking [see Figs. 4(c) and 4(d)], from which we conclude that slip of the folded defect into the surrounding layer is inhibited due to friction.

The constant formation energy can be understood with reference to the relaxed structures, shown in Fig. 4(a). As extra material is added, it is accommodated by increasing the length of the prismatic sheet bounded by the highly curved regions, while the curved regions remain approximately the same. This demonstrates that for both bulk and surface ripples, curvature 
(a) $n=3$

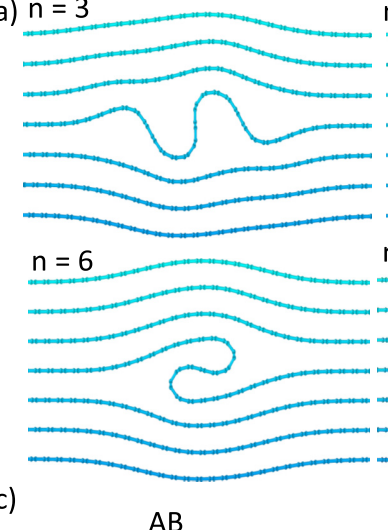

$n=4$

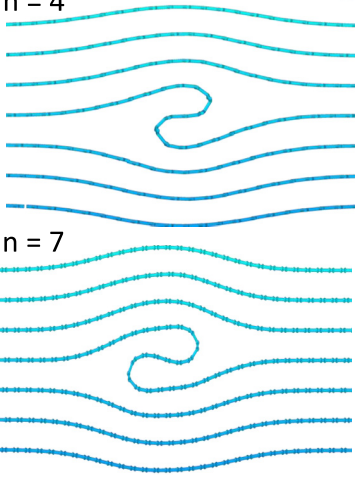

$\mathrm{n}=5$

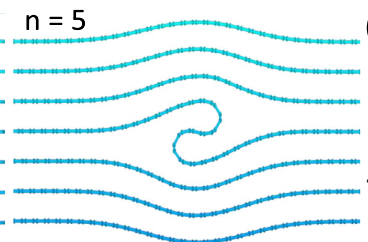

$\mathrm{n}=8$

$A B$

(d)

$A B$

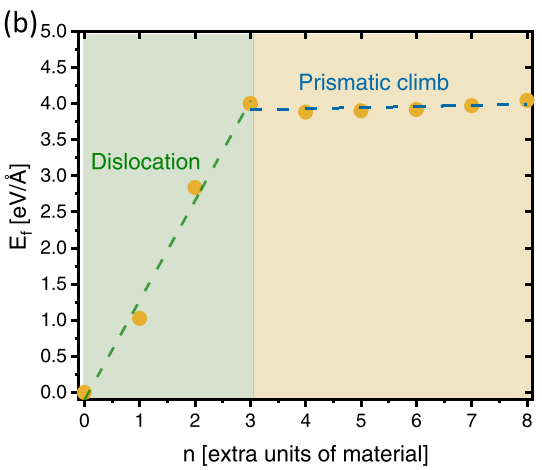

$n=6$

$A B$
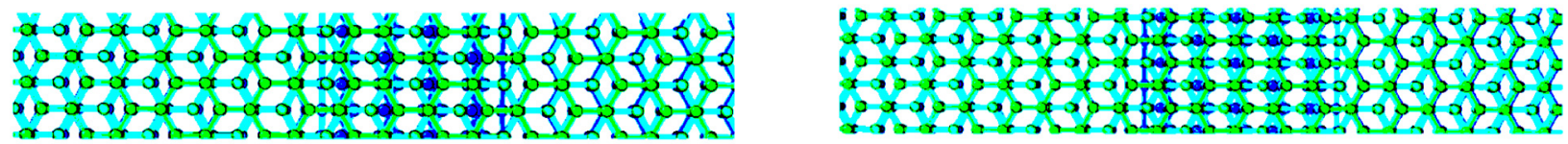

FIG. 4. (a) Single-layer pileup in bulk graphite for different values of $n$, resulting in the "ruck and tuck" structure with increasing material. (b) Bulk ripplocation formation energy vs $n$. (c) and (d) Plan view above a ruck and tuck defect, demonstrating that Bernal stacking is recovered in the material surrounding the folded defect core.

can essentially be seen as the mechanism of dislocation climb for basal dislocations in layered materials. This proceeds unconstrained on the surface, while in bulk additional material is accommodated simply by growing the flat, perfectly stacked prismatic layer.

Dislocation pileups on multiple adjacent layers in bulk graphite have also been considered through the incorporation of extra material to a number of adjacent layers in a large bulk simulation cell. These structures are the corresponding bulk defect to the finite-thickness surface ripplocations. Upon relaxation we find structures which are quite distinct from the single-layer ruck and tuck, which are more similar to bulk twin boundaries [55], demonstrating multilayer curvature and the formation of voids adjacent to the compressed region, some representative structures of which are shown in Fig. 5(a). The distinction between the single and multilayer defects can be understood through consideration of the bending energy for multilayer graphene, which scales linearly for small thicknesses [56].
The formation energy per Burgers vector per layer for these defects is shown in Fig. 5(b), which we see results in even lower energy per $n$ than the single-layer ruck and tuck. The ruck and tuck can then be seen as a limiting case where compression occurs on only one layer. It is therefore unsurprising that the structure of irradiated HOPG generally involves larger-scale curvature, as the higher bending energy inhibits the ruck and tuck for the lower-energy, finitethickness bulk ripplocations. However, the presence of both the ruck and tuck defect as well as large-scale bending can be taken as evidence for dislocation pileup as the deformation mode of irradiated HOPG. Furthermore, all of the bulk structures simulated in this work essentially demonstrate basal climb, i.e., $c$-axis expansion to release in-plane compression resulting from many basal dislocations.

\section{TWO-DIMENSIONAL RIPPLES}

MD simulations also permit calculations of other large structures which are prohibitive for DFT, such as the two- (a)

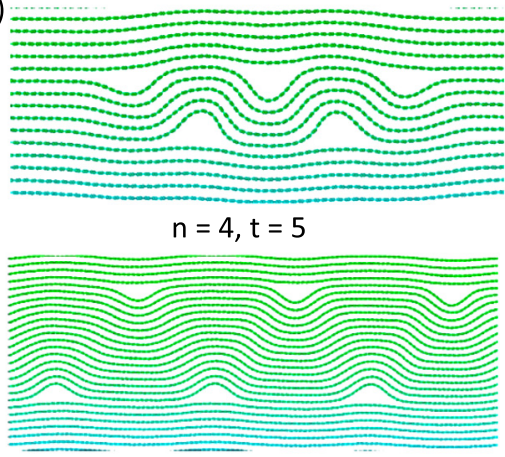

$\mathrm{n}=4, \mathrm{t}=15$

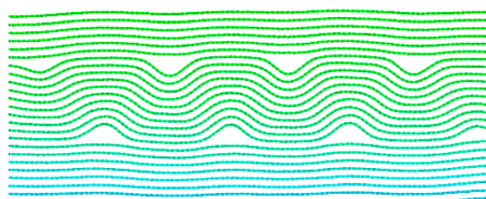

$\mathrm{n}=4, \mathrm{t}=10$

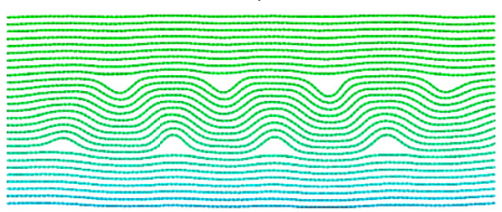

$\mathrm{n}=5, \mathrm{t}=10$

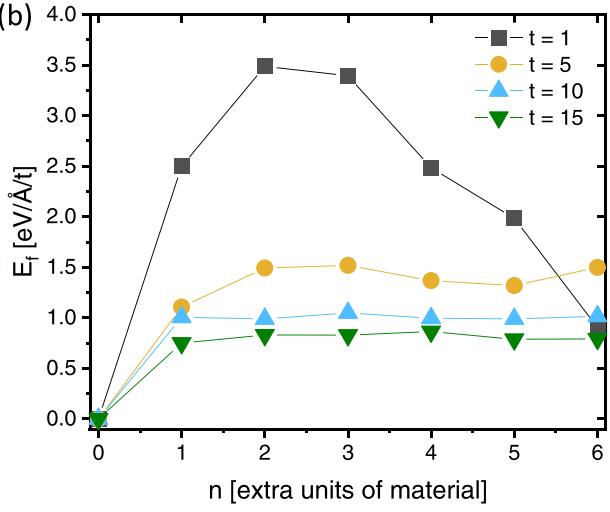

FIG. 5. (a) Multilayer (finite thickness, $t$ ) dislocation pileups. (b) Energy per length of additional material of multilayer dislocation pileups. 


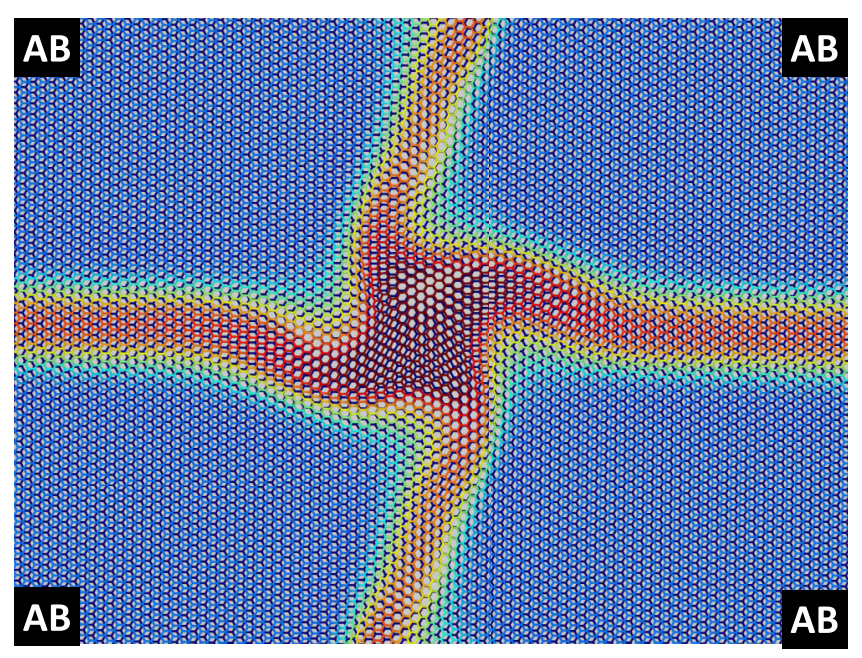

FIG. 6. Two-dimensional ripplocation junction on the graphite (0001) surface. Carbon atoms are color-coded according to height. Blue sections are flat $\mathrm{AB}$-stacked regions. The ripples' height is around $1 \mathrm{~nm}$, red sections.

dimensional ripplocation junction shown in Fig. 6. This has been created by inserting material along both the armchair and zigzag directions on the surface of a bilayer cell. The pictured cell, which has an initial Burgers vector $\mathbf{b}=\left(b_{a c}, b_{z z}\right)=$ $(2,1)$, results in the formation of a ripplocation junction, where perpendicular dislocation lines meet at an AA-stacked dislocation node. It is noteworthy that this type of structure is strikingly similar to experimental TEM images of irradiated HOPG surfaces [21,22], and in this context our large-scale simulations again provide evidence of dislocation pileup as the likely deformation mode of irradiated HOPG. We leave further, systematic investigation of these two-dimensional defects to future work.

\section{DISCUSSION}

The application of classical mechanics to the nanoscale buckling of two-dimensional monolayers has typically proven difficult [57,58]. In this work, we have found that minor modification of classical expressions gives exceptional agreement to DFT calculations. The energy scaling, Eq. (2), is based only on classical expressions for clamped elastica and is, therefore, directly applicable to any inextensible layered material, through simple modification of the $\alpha$ sublinearity parameter. This reinforces the idea that ripplocations are a general concept applicable at a variety of length scales, since we have considered only monolayer bending and adhesion.

The exceptionally low sublinear factors, in the range $\alpha \approx$ $0.1-0.18$, in comparison to a sheet under the influence of gravity, helps to explain the exaggerated tendency of epi- taxial monolayers to wrinkle and ripple. Our work therefore indicates that the most important property in the wrinkling of epitaxially grown monolayers is the coefficient of friction between the monolayer and the substrate. This can explain, for example, the high density of wrinkle formation in graphene adsorbed on the the highly incommensurate $\mathrm{Cu}(100)$ and $\mathrm{Cu}(110)$ surfaces [59] in comparison to the almostcommensurate $\mathrm{Cu}(111)$ face. It is therefore noteworthy that the ripplocation formation energy is close to the observed experimental energy barrier for wrinkle nucleation on $\operatorname{Ir}(100)$ substrates [60,61].

In all cases, buckling of the ripplocation can be seen as a type of climb mechanism for basal dislocations. Our work therefore has important implications for the irradiationinduced deformation of HOPG. Under irradiation, HOPG and even single graphene sheets will shrink, which very quickly leads to the formation of basal dislocations. The very low Peierls barrier in layered materials ensures that the basal dislocation core is very diffuse and easy to move, which readily allows dislocation pileups [50,54]. Under this compression, our work confirms that these dislocation pileups will result in delaminated, curved regions of microstructure rather than flat superdislocation cores. This suggests that essentially a process of subsurface wrinkling is implicated in the pronounced $c$ axis expansion of irradiated HOPG samples, with increasing compression resulting in the formation of ripplocation arrays, voids, ruck and tucks, and highly curved regions. It is then unsurprising that there are substantial similarities between our simulations and experiment. Under heavy ion bombardment, in-plane dislocations are nucleated, which evolve into rippled "kink boundaries." These two-dimensional dislocation complexes are almost identical to our simulations of surface ripplocation arrays.

There is also substantial experimental evidence for the formation of the single-layer ruck and tuck in irradiated graphite [8,15-20]. In addition, our multilayer simulations are similar to the regions of curvature and voids, which have recently been found in irradiated HOPG [62], again demonstrating damage due to compression and pileup. In summary, this correspondence implicates the compression-induced dislocation pileup and rippling of graphene monolayers as a concise and appealing explanation of the irradiation-induced deformation of single-crystal graphite.

\section{ACKNOWLEDGMENTS}

J.G.M. was supported by UK EPSRC Grant No. EP/R005745/1, Mechanisms of Retention and Transport of Fission Products in Virgin and Irradiated Nuclear Graphite. K.J. and P.M. gratefully acknowledge funding from EDF Energy. The authors acknowledge the use of the HPC Midlands+ facility, funded by EPSRC Grant No. EP/P020232/1, as part of the HPC Midlands+ consortium.
[1] F. C. Frank and W. T. Read, Multiplication processes for slow moving dislocations, Phys. Rev. 79, 722 (1950).

[2] F. Frank, Crystal dislocations: Elementary concepts and definitions, The London, Edinburgh, and Dublin Philosophical Magazine and Journal of Science 42, 809 (1951).
[3] F. C. Frank and J. H. van der Merwe, One-dimensional dislocations. I. Static theory, Proc. R. Soc. London, Ser. A 198, 205 (1949).

[4] A. Kushima, X. Qian, P. Zhao, S. Zhang, and J. Li, Ripplocations in van der Waals layers, Nano Lett. 15, 1302 (2015). 
[5] A. Alaferdov, R. Savu, M. Canesqui, Y. Kopelevich, R. da Silva, N. Rozhkova, D. Pavlov, Y. Usov, G. de Trindade, and S. Moshkalev, Ripplocation in graphite nanoplatelets during sonication assisted liquid phase exfoliation, Carbon 129, 826 (2018).

[6] M. Barsoum and G. Tucker, Deformation of layered solids: Ripplocations not basal dislocations, Scr. Mater. 139, 166 (2017).

[7] J. Aslin, E. Mariani, K. Dawson, and M. W. Barsoum, Ripplocations provide a new mechanism for the deformation of phyllosilicates in the lithosphere, Nat. Commun. 10, 686 (2019).

[8] M. W. Barsoum, Ripplocations: A progress report, Front. Mater. 7, 146 (2020).

[9] M. W. Barsoum, X. Zhao, S. Shanazarov, A. Romanchuk, S. Koumlis, S. J. Pagano, L. Lamberson, and G. J. Tucker, Ripplocations: A universal deformation mechanism in layered solids, Phys. Rev. Mater. 3, 013602 (2019).

[10] F. Zheng, Q. H. Thi, L. W. Wong, Q. Deng, T. H. Ly, and J. Zhao, Critical stable length in wrinkles of two-dimensional materials, ACS Nano 14, 2137 (2020).

[11] Y.-S. No, J. H. Lee, B. H. Park, and J. S. Choi, Ripples, wrinkles, and crumples in folded graphene, J. Korean Phys. Soc. 76, 985 (2020).

[12] M. Tripathi, F. Lee, A. Michail, D. Anestopoulos, J. G. McHugh, S. P. Ogilvie, M. J. Large, A. A. Graf, P. J. Lynch, J. Parthenios, K. Papagelis, S. Roy, M. A. S. R. Saadi, M. M. Rahman, N. M. Pugno, A. A. K. King, P. M. Ajayan, and A. B. Dalton, Structural defects modulate electronic and nanomechanical properties of 2D materials, ACS Nano 15, 2520 (2021)

[13] W. Zhu, T. Low, V. Perebeinos, A. A. Bol, Y. Zhu, H. Yan, J. Tersoff, and P. Avouris, Structure and electronic transport in graphene wrinkles, Nano Lett. 12, 3431 (2012).

[14] S. Deng and V. Berry, Wrinkled, rippled and crumpled graphene: An overview of formation mechanism, electronic properties, and applications, Mater. Today 19, 197 (2016).

[15] A. Asthana, Y. Matsui, M. Yasuda, K. Kimoto, T. Iwata, and $\mathrm{K}$. Ohshima, Investigations on the structural disordering of neutron-irradiated highly oriented pyrolytic graphite by $\mathrm{x}$-ray diffraction and electron microscopy, J. Appl. Crystallogr. 38, 361 (2005).

[16] J. Eapen, R. Krishna, T. D. Burchell, and K. L. Murty, Early damage mechanisms in nuclear grade graphite under irradiation, Mater. Res. Lett. 2, 43 (2013).

[17] S. Muto and T. Tanabe, Fragmentation of graphite crystals by electron irradiation at elevated temperatures, J. Electron Microsc. 48, 519 (1999).

[18] S. Johns, L. He, K. Bustillo, W. E. Windes, R. Ubic, and C. Karthik, Fullerene-like defects in high-temperature neutronirradiated nuclear graphite, Carbon 166, 113 (2020).

[19] S. Johns, L. He, J. J. Kane, W. E. Windes, R. Ubic, and C. Karthik, Experimental evidence for 'buckle, ruck and tuck' in neutron irradiated graphite, Carbon 159, 119 (2020).

[20] C.-T. Pan, J. A. Hinks, Q. M. Ramasse, G. Greaves, U. Bangert, S. E. Donnelly, and S. J. Haigh, In-situ observation and atomic resolution imaging of the ion irradiation induced amorphisation of graphene, Sci. Rep. 4, 6334 (2014).

[21] J. Hinks, S. Haigh, G. Greaves, F. Sweeney, C. Pan, R. Young, and S. Donnelly, Dynamic microstructural evolution of graphite under displacing irradiation, Carbon 68, 273 (2014).
[22] J. A. Hinks, G. Greaves, S. J. Haigh, C.-T. Pan, and S. E. Donnelly, Kink band formation in graphite under ion irradiation at 100 and 298 K, Mater. Trans. 55, 447 (2014).

[23] J. Gruber, A. C. Lang, J. Griggs, M. L. Taheri, G. J. Tucker, and M. W. Barsoum, Evidence for bulk ripplocations in layered solids, Sci. Rep. 6, 33451 (2016).

[24] A. Ostadhossein, A. Rahnamoun, Y. Wang, P. Zhao, S. Zhang, V. H. Crespi, and A. C. T. van Duin, ReaxFF: Reactive forcefield study of molybdenum disulfide $\left(\mathrm{MoS}_{2}\right)$, J. Phys. Chem. Lett. 8, 631 (2017).

[25] S. Dai, Y. Xiang, and D. J. Srolovitz, Structure and energetics of interlayer dislocations in bilayer graphene, Phys. Rev. B 93, 085410 (2016).

[26] A. N. Kolmogorov and V. H. Crespi, Registry-dependent interlayer potential for graphitic systems, Phys. Rev. B 71, 235415 (2005).

[27] A. M. Popov, I. V. Lebedeva, A. A. Knizhnik, Y. E. Lozovik, and B. V. Potapkin, Molecular dynamics simulation of the selfretracting motion of a graphene flake, Phys. Rev. B 84, 245437 (2011).

[28] M. Wen, S. Carr, S. Fang, E. Kaxiras, and E. B. Tadmor, Dihedral-angle-corrected registry-dependent interlayer potential for multilayer graphene structures, Phys. Rev. B 98, 235404 (2018).

[29] O. Hod, Interlayer interactions in low-dimensional layered hetero-structures: Modeling and applications, in Handbook of Materials Modeling (Springer International Publishing, 2018), pp. 1-25.

[30] P. Giannozzi et al., QUANTUM ESPRESSO: A modular and open-source software project for quantum simulations of materials, J. Phys.: Condens. Matter 21, 395502 (2009).

[31] P. Giannozzi et al., Advanced capabilities for materials modelling with Quantum ESPRESSO, J. Phys.: Condens. Matter 29, 465901 (2017).

[32] K. F. Garrity, J. W. Bennett, K. M. Rabe, and D. Vanderbilt, Pseudopotentials for high-throughput DFT calculations, Comput. Mater. Sci. 81, 446 (2014).

[33] M. Nekovee, W. M. C. Foulkes, and R. J. Needs, Quantum Monte Carlo investigations of density functional theory of the strongly inhomogeneous electron gas, Phys. Rev. B 68, 235108 (2003)

[34] E. Mostaani, N. D. Drummond, and V. I. Fal'ko, Quantum Monte Carlo Calculation of the Binding Energy of Bilayer Graphene, Phys. Rev. Lett. 115, 115501 (2015).

[35] F. Tran, L. Kalantari, B. Traoré, X. Rocquefelte, and P. Blaha, Nonlocal van der Waals functionals for solids: Choosing an appropriate one, Phys. Rev. Mater. 3, 063602 (2019).

[36] I. Mosyagin, D. Gambino, D. G. Sangiovanni, I. A. Abrikosov, and N. M. Caffrey, Effect of dispersion corrections on $a b$ initio predictions of graphite and diamond properties under pressure, Phys. Rev. B 98, 174103 (2018).

[37] J. G. McHugh, K. Jolley, and P. Mouratidis, Ab-initio calculations of fission product diffusion on graphene, J. Nucl. Mater. 533, 152123 (2020).

[38] R. Zacharia, H. Ulbricht, and T. Hertel, Interlayer cohesive energy of graphite from thermal desorption of polyaromatic hydrocarbons, Phys. Rev. B 69, 155406 (2004).

[39] W. Wang, S. Dai, X. Li, J. Yang, D. J. Srolovitz, and Q. Zheng, Measurement of the cleavage energy of graphite, Nat. Commun. 6, 7853 (2015). 
[40] S. Plimpton, Fast parallel algorithms for short-range molecular dynamics, J. Comput. Phys. 117, 1 (1995).

[41] M. Wen and E. B. Tadmor, Hybrid neural network potential for multilayer graphene, Phys. Rev. B 100, 195419 (2019).

[42] E. B. Tadmor, R. S. Elliott, J. P. Sethna, R. E. Miller, and C. A. Becker, The potential of atomistic simulations and the knowledgebase of interatomic models, JOM 63, 17 (2011).

[43] I. V. Lebedeva, A. A. Knizhnik, A. M. Popov, Y. E. Lozovik, and B. V. Potapkin, Interlayer interaction and relative vibrations of bilayer graphene, Phys. Chem. Chem. Phys. 13, 5687 (2011).

[44] L. A. Girifalco and M. Hodak, Van der Waals binding energies in graphitic structures, Phys. Rev. B 65, 125404 (2002).

[45] See Supplemental Material at http://link.aps.org/supplemental/ 10.1103/PhysRevB.103.195436 for additional details on interlayer atomic potentials, fixed layer approximation, bond length and strain across a ripplocation, and graphene/Cu simulations.

[46] J. S. Alden, A. W. Tsen, P. Y. Huang, R. Hovden, L. Brown, J. Park, D. A. Muller, and P. L. McEuen, Strain solitons and topological defects in bilayer graphene, Proc. Natl. Acad. Sci. USA 110, 11256 (2013).

[47] J. Lin, W. Fang, W. Zhou, A. R. Lupini, J. C. Idrobo, J. Kong, S. J. Pennycook, and S. T. Pantelides, AC/AB stacking boundaries in bilayer graphene, Nano Lett. 13, 3262 (2013).

[48] B. Butz, C. Dolle, F. Niekiel, K. Weber, D. Waldmann, H. B. Weber, B. Meyer, and E. Spiecker, Dislocations in bilayer graphene, Nature (London) 505, 533 (2013).

[49] I. V. Lebedeva, A. V. Lebedev, A. M. Popov, and A. A. Knizhnik, Dislocations in stacking and commensurateincommensurate phase transition in bilayer graphene and hexagonal boron nitride, Phys. Rev. B 93, 235414 (2016).

[50] R. H. Telling and M. I. Heggie, Stacking fault and dislocation glide on the basal plane of graphite, Philos. Mag. Lett. 83, 411 (2003).

[51] D. Vella, A. Boudaoud, and M. Adda-Bedia, Statics and Inertial Dynamics of a Ruck in a Rug, Phys. Rev. Lett. 103, 174301 (2009).

[52] J. M. Kolinski, P. Aussillous, and L. Mahadevan, Shape and Motion of a Ruck in a Rug, Phys. Rev. Lett. 103, 174302 (2009).
[53] Q. Lu, M. Arroyo, and R. Huang, Elastic bending modulus of monolayer graphene, J. Phys. D 42, 102002 (2009).

[54] M. Heggie, I. Suarez-Martinez, C. Davidson, and G. Haffenden, Buckle, ruck and tuck: A proposed new model for the response of graphite to neutron irradiation, J. Nucl. Mater. 413, 150 (2011).

[55] A. P. Rooney, Z. Li, W. Zhao, A. Gholinia, A. Kozikov, G. Auton, F. Ding, R. V. Gorbachev, R. J. Young, and S. J. Haigh, Anomalous twin boundaries in two dimensional materials, Nat. Commun. 9, 3597 (2018).

[56] E. Han, J. Yu, E. Annevelink, J. Son, D. A. Kang, K. Watanabe, T. Taniguchi, E. Ertekin, P. Y. Huang, and A. M. van der Zande, Ultrasoft slip-mediated bending in few-layer graphene, Nat. Mater. 19, 305 (2019).

[57] L. Tapasztó, T. Dumitrică, S. J. Kim, P. Nemes-Incze, C. Hwang, and L. P. Biró, Breakdown of continuum mechanics for nanometre-wavelength rippling of graphene, Nat. Phys. 8, 739 (2012).

[58] W. Bao, F. Miao, Z. Chen, H. Zhang, W. Jang, C. Dames, and C. N. Lau, Controlled ripple texturing of suspended graphene and ultrathin graphite membranes, Nat. Nanotechnol. 4, 562 (2009).

[59] B. Deng, Z. Pang, S. Chen, X. Li, C. Meng, J. Li, M. Liu, J. Wu, Y. Qi, W. Dang, H. Yang, Y. Zhang, J. Zhang, N. Kang, H. Xu, Q. Fu, X. Qiu, P. Gao, Y. Wei, Z. Liu et al., Wrinkle-free singlecrystal graphene wafer grown on strain-engineered substrates, ACS Nano 11, 12337 (2017).

[60] H. Hattab, A. T. N’Diaye, D. Wall, C. Klein, G. Jnawali, J. Coraux, C. Busse, R. van Gastel, B. Poelsema, T. Michely, F.-J. M. zu Heringdorf, and M. H. von Hoegen, Interplay of wrinkles, strain, and lattice parameter in graphene on iridium, Nano Lett. 12, 678 (2012).

[61] A. T. N'Diaye, R. van Gastel, A. J. Martínez-Galera, J. Coraux, H. Hattab, D. Wall, F.-J. M. zu Heringdorf, M. H. von Hoegen, J. M. Gómez-Rodríguez, B. Poelsema, C. Busse, and T. Michely, In situ observation of stress relaxation in epitaxial graphene, New J. Phys. 11, 113056 (2009).

[62] S. Johns, T. Poulsen, J. J. Kane, W. E. Windes, R. Ubic, and C. Karthik, Formation of carbon nanostructures in nuclear graphite under high-temperature in situ electron-irradiation, Carbon 143, 908 (2019). 